\title{
A multi-centre analysis of cardiac surgery referral patterns and resource allocation during the COVID-19 pandemic
}

\author{
Damian Balmforth ${ }^{1}$, Ana Lopez-Marco ${ }^{1}$, Martin Yates ${ }^{1}$, Benjamin Adams $^{1}$, Alex Cale ${ }^{2}$, \\ Reuben Jeganathan ${ }^{3}$, Indu Deglurkar ${ }^{4}$, Bilal Kirmani ${ }^{5}$, Mazyar Kanani ${ }^{6}$, Giovanni \\ Mariscalco $^{7}$, Saleem Jahangeer ${ }^{8}$, George Krasopoulos ${ }^{9}$, Mohamed Sherif ${ }^{10}$, Yasir Ahmed ${ }^{11}$, \\ Aung $\mathrm{Oo}^{1}$, and Julie Sanders ${ }^{12}$ \\ ${ }^{1}$ Saint Bartholomew's Hospital \\ ${ }^{2}$ Hull University Teaching Hospitals NHS Trust \\ ${ }^{3}$ Royal Victoria Hospital \\ ${ }^{4}$ University Hospital of Wales Healthcare NHS Trust \\ ${ }^{5}$ Liverpool Heart and Chest Hospital NHS Foundation Trust \\ ${ }^{6}$ James Cook University Hospital \\ ${ }^{7}$ University Hospitals of Leicester NHS Trust \\ ${ }^{8}$ Manchester University NHS Foundation Trust \\ ${ }^{9}$ Oxford University Hospitals NHS Foundation Trust \\ ${ }^{10}$ Sheffield Teaching Hospitals NHS Foundation Trust \\ ${ }^{11}$ Swansea Bay University Health Board \\ ${ }^{12}$ St Bartholomew's Hospital
}

September 25, 2021

\begin{abstract}
Background and aims: The COVID-19 pandemic caused a dramatic shift in the provision of cardiac surgical services in the United Kingdom (UK) with all elective surgery suspended. We sought to explore referral patterns, changes in clinical decision making and resource allocation to adult cardiac surgical services in the UK during the first wave of the pandemic. Methods: Data from 11 UK centres on referrals and available health resources (operating theatre and bed capacity) for urgent or emergency adult cardiac surgery between the 1st March 2020 and the 1st August 2020 was collated, and securely transferred to the lead centre for analysis. Results: 1113 patients were referred for cardiac surgery over the study period. Following UK lockdown in March 2020 the number of referrals initially fell to $39 \%$ of pre-lockdown levels before recovering to $211 \%$ of that seen prior to the pandemic. A change in treatment strategies was observed with a trend towards deferring surgery entirely or favouring less invasive, non-surgical treatments. At the peak of the pandemic in April 2020, theatre availability and bed capacity fell to $26 \%$ and $54 \%$ of pre-lockdown levels, respectively. Provision for emergency surgery was maintained throughout at 1 to 2 emergency lists per unit weekly. Conclusion: During the first wave of the UK COVID-19 pandemic cardiac surgical operative activity dropped acutely before increasing over the next four months. Despite this drop, provision for emergency surgery was retained throughout. In the event of further waves of COVID-19 pandemic, maintaining essential cardiac surgical services should be prioritised.
\end{abstract}

\section{Hosted file}

Changes in cardiac surgery referral patterns J0CS V1.docx available at https://authorea. com/users/327298/articles/539158-a-multi-centre-analysis-of-cardiac-surgery-referral- 
patterns-and-resource-allocation-during-the-covid-19-pandemic
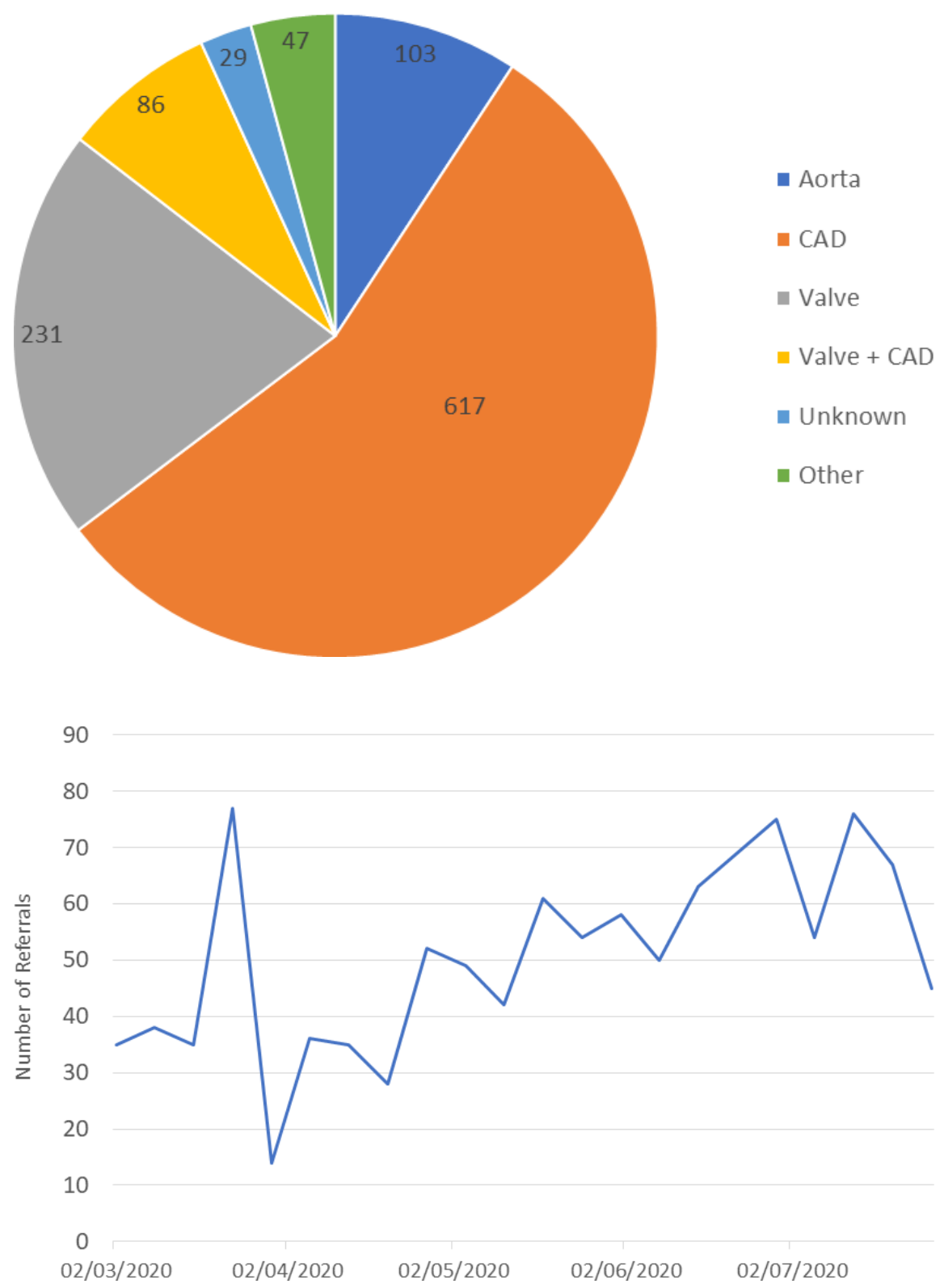

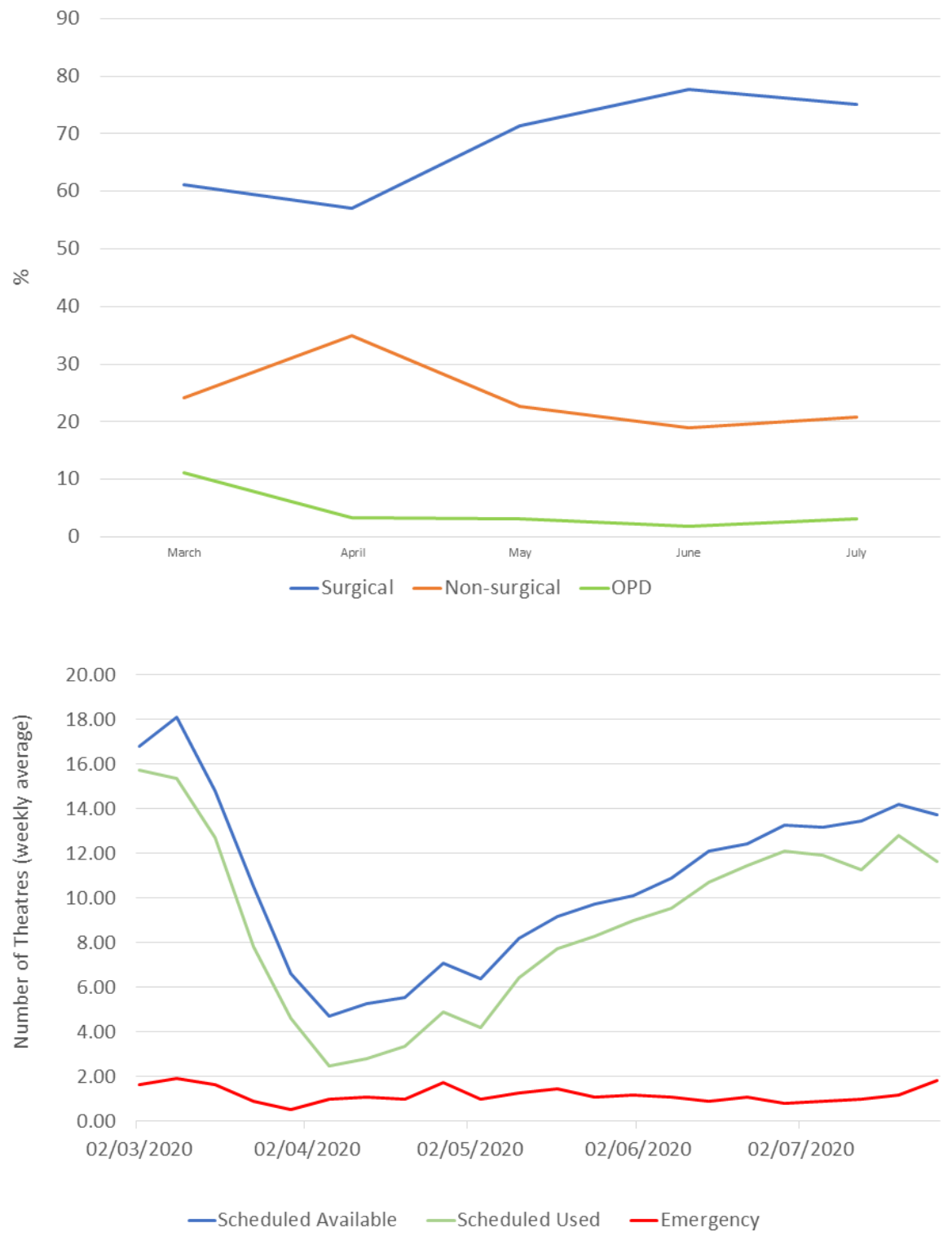


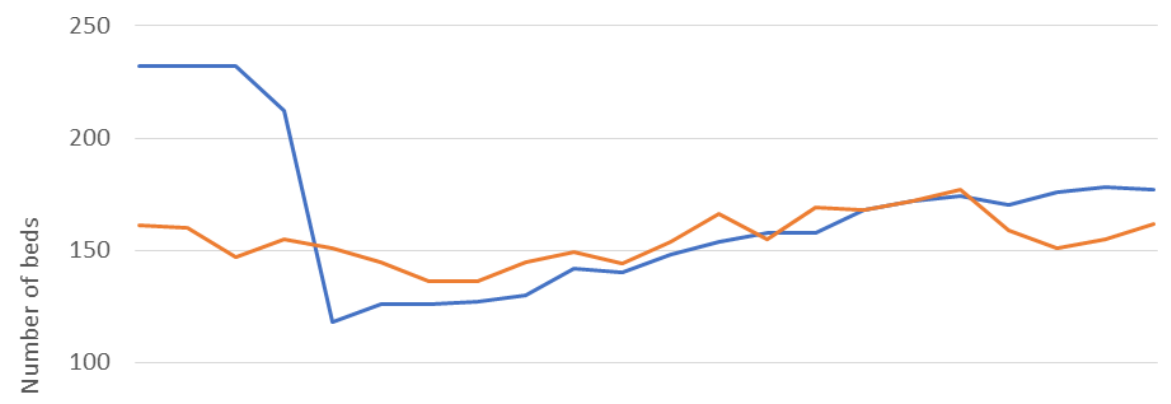

50

0

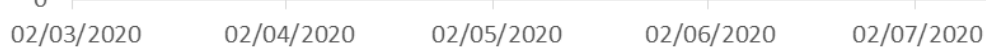

—Total Ward Beds _Total ITU Beds

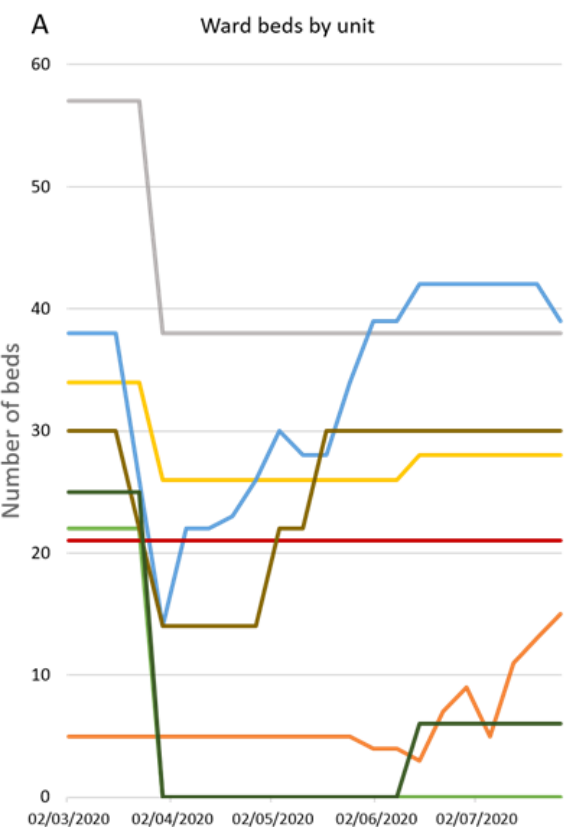

B ITU bed by unit

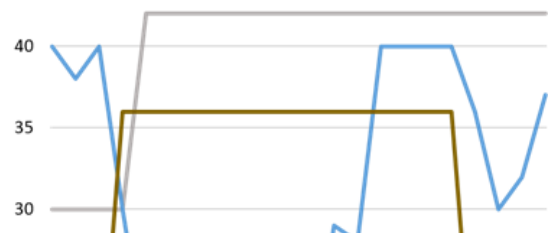

25

20

15

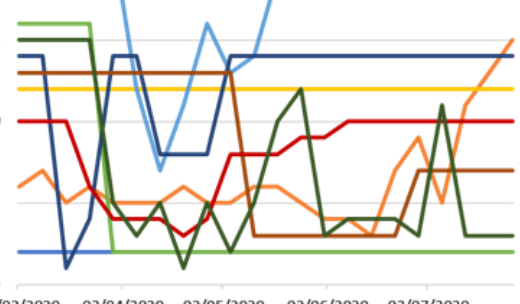

$\begin{array}{lllll}02 / 03 / 2020 & 02 / 04 / 2020 & 02 / 05 / 2020 & 02 / 06 / 2020 & 02 / 07 / 2020\end{array}$

$\begin{array}{lllll}02 / 03 / 2020 & 02 / 04 / 2020 & 02 / 05 / 2020 & 02 / 06 / 2020 & 02 / 07 / 2020\end{array}$ 